\title{
The Effect of Diazepam Upon the Development of Hypertension in the Spontaneously Hypertensive Rat
}

\author{
RICHARD M. SCHIEKEN \\ Department of Pediatrics, Division of Pediatric Cardiology, University of Iowa Hospitals and Clinics, Iowa City, Iowa \\ $U S A$
}

\begin{abstract}
Summary
The long term antihypertensive effects of the chronic administration of diazepam (D) beginning in the newborn period to spontaneously hypertensive rat (SHR) is the subject of this study. The following treatment groups were investigated: SHR-D $=$ SHR treated with diazepam; SHR-V $=$ SHR treated with diazepam vehicle; WKY-D = Wistar Kyoto control rat (WKY) treated with diazepam; WKY-V = WKY treated with vehicle. Treatment was begun on the 1st day of life. Injections of the following agents were made during hindquarter perfusion: norephinephrine bitartrate, epinephrine hydrochloride, angiotensin-II, barium chloride, and acetylcholine. SHR-V had persistent, progressive hypertension. The blood pressure of SHR-D rose slowly, but did attain levels higher than pressures of either WKY groups. In response to diazepam, the vascular resistance of SHR-D was reduced in comparison to SHR-V. SHR showed significantly greater vascular responses to catecholamines as compared to WKY. These enhanced vascular responses were reduced, but not abolished, by diazepam. When diazepam was administered to adult SHR with established hypertension, the blood pressure was not reduced. No decrease in vascular reactivity was found. In conclusion, treatment from birth with diazepam exerts a profound effect upon the development of high blood pressure and elevated vascular resistance in SHR. Only the young prehypertensive animals treated chronically were responsive to diazepam. The major effect of diazepam may be mediated via generalized depression of vascular smooth muscle responsiveness.
\end{abstract}

\section{Speculation}

Early pharmacologic intervention in young animals, genetically predisposed to severe hypertension, blunts the expression of the disease. This suggests that prehypertensive animals have alterations that can be pharmacologically modified. Vascular smooth muscle may be the common pathway of action for these agents.

The SHR developed by Okamoto and Aoki (17) is an excellent model for the investigation of the pathogenesis of a hypertensive state. Though suckling SHR are normotensive, hypertension is apparent soon after weaning. (13) The mechanisms responsible for the development of increased blood pressure in SHR are unclear.

Many studies suggest that an abnormal neurogenic stimulus may initiate or augment hypertension. However, the evidence for a central nervous system contribution remains controversial. Elevated hindquarter vascular resistance has been reported both to be relieved by (16), and not relieved by (14), lumbar sympathetic nerve section. Deafferentiation of the baroreceptors produces severe hypertension (21). In addition, after sinoaortic deafferentiation, the blood pressure becomes extremely labile during environmental stimulation (3). Studies of sympathetic nerve activity suggest that central sympathetic centers, uninhibited by baroreceptor influence, may become active in young SHR and participate in the development of hypertension (9).

Hypertension can be initiated by environmental stress. Presumably this effect is mediated by increased sympathetic drive. Various stresses which include crowding (7), noise (15), and stressful learning situations (8) can cause hypertension. By raising SHR in a quiet, dark environment, thereby removing major environmental stimuli, the development of hypertension in SHR is retarded (10).

Both because chronic stress is known to augment hypertension (24) and because decreased environmental stimuli decrease blood pressure $(10)$, we tested the effects of the chronic administration of the tranquilizing agent, diazepam, upon the development of hypertension in SHR. The long term antihypertensive effects of the chronic administration of diazepam to young SHR is the subject of this report.

\section{METHODS}

SHR were obtained from an inbred colony maintained in our laboratory. The SHR reported were the 5th through the 9th generation raised in these facilities and correspond respectively to the $22 \mathrm{nd}$ to the 26th generation, accurately traced back to the original pairing resulting in the derivation of this strain. Control rats were obtained from a colony of WKY that are bred and maintained under the same conditions which applied to the SHR. This strain is descended directly from the colony from which the SHR were isolated and are appropriate controls for the SHR strain. Animals were randomly assigned to groups on the lst day of life and identified by marking the coat with colored ink. The animals were weaned on the 21 st day of life.

Systolic pressure was measured three times weekly in the unanesthetized state by modification of the tail plethysmograph method described by Fridman and Freed (5) using an automated cuff inflator pulse detection system manufactured by Technilab Instruments (Pequananock, NJ). Before pressure measurements the animals were warmed in a cage with an air temperature of $35^{\circ} \mathrm{C}$ for $5 \mathrm{~min}$. The data presented are the averages per wk of weight, heart rate, and blood pressure recorded three times weekly beginning after the animals attained the weight of $28 \mathrm{~g}$.

The following treatment groups of 10 animals each were studied: SHR-D - spontaneously hypertensive rat treated with diazepam that was generously supplied by Dr. Leonard Cook, Roche Laboratories, Nutley, NJ (composition of vehicle includes propylene glycol, ethyl alcohol, sodium benzoate and benzyl alcohol) $1 \mathrm{mg}$ / kg subcutaneously (a dose which produced transient sedation, but no effect upon normal eating, drinking, or growth); SHR-Vspontaneously hypertensive rat given diazepam vehicle, also supplied by Dr. Cook; WKY-D-WKY given diazepam $1 \mathrm{mg} / \mathrm{kg}$ subcutaneously; and WKY-V-WKY given diazepam vehicle. The vehicle was administered in equivalent volume. Treatment was begun on the lst day of life after randomization and continued 
7 days/wk for 12 wk. Regardless of treatment group assignment, the rat pups were allowed to suckle their own mother.

After approximately $12 \mathrm{wk}$ of treatment, or when the SHR-V animals were definitely hypertensive, hindquarter perfusion experiments were begun using the technique of Brody et al. (1). Experiments were performed $24 \mathrm{hr}$ after the last dosage. The rats were anesthetized using sodium pentobarbital $30 \mathrm{mg} / \mathrm{kg}$. A midline cervical incision was made and a tracheal cannula was inserted. The carotid artery was cannulated for continuous recording of mean systemic arterial pressure using a Statham P23A pressure transducer and a Beckman Type RM recorder. The aorta was doubly cannulated, and the blood from the proximal aorta diverted through Tygon tubing inserted into isolated hindquarter above the level of the bifurcation of the abdominal aorta. Constant flow was maintained by a Sigmamotor pump inserted within the circuit. At constant flow, changes in perfusion pressure reflected proportional changes in vascular resistance. The blood flow to the hindquarter was adjusted so that the perfusion pressure closely approximated the systemic arterial pressure measured from the carotid artery. Drugs were injected intraarterially into a rubber cuff just proximal to the caudal cannulation site. The animal was anticoagulated using sodium heparin $5 \mathrm{mg} / \mathrm{kg}$. A period of $15 \mathrm{~min}$ was allowed for anticoagulant action.

Injections of the following agents were made in volumes of 5 6 microliters using a $50 \mu \mathrm{l}$ syringe (Hamilton Company Incorporated): norepinephrine bitartrate $0.06 \mu \mathrm{g} / \mathrm{kg}$, epinephrine hydrochloride $0.06 \mu \mathrm{g} / \mathrm{kg}$, angiotensin II $0.5 \mu \mathrm{g} / \mathrm{kg}$, barium chloride 1.0 $\mathrm{mg} / \mathrm{kg}$, acetycholine bromide $25 \mu \mathrm{g} / \mathrm{kg}$ (given over $1 \mathrm{~min}$ ). In addition, the lumbar sympathetic nerves were exposed and isolated. The nerves were electrically stimulated bilaterally at L-3 through a small bipolar electrode with square waves generated from a Grass stimulator. The electrical stimulus was 10 volts, with a pulse duration of $2 \mathrm{msec}$, at frequencies of 10 and $20 \mathrm{~Hz}$. Changes in vascular resistance were calculated from changes in pressure in the isolated hindquarter.

An additional group of five adult SHR with established hypertension received similar daily doses of diazepam for 6 days. Preand posttreatment blood pressures and pulse rates were measured. After the 6th treatment day, $24 \mathrm{hr}$ after the last dosage, vascular resistance studies were performed.

Data were analyzed using Tukey's multiple comparison procedure for analysis of variance (22).

\section{RESULTS}

Figure 1 illustrates the mean and SE of the tail cuff blood pressures of the four groups of animals. SHR-V had persistent, progressive hypertension. During the 6th and 7th wk, SHR-D remained normotensive. However, by the 8th week the blood pressure of SHR-D rose to levels higher than the pressures of both WKY-D and WKY-V. No differences were observed throughout the experiment between the blood pressures of WKY-D versus WKY-V.

Table 1 shows the body wt, heart rate, initial vascular resistance, and mean arterial pressure of the four groups of animals. The initial vascular resistance and systemic arterial pressure of SHR$D$ were reduced compared to SHR-V $(P<0.01)$. Despite this reduction, the vascular resistance and systemic pressure of SHR$D$ remained higher than the WKY groups $(P<0.05)$. No effects upon vascular resistance were observed in WKY-D. No significant differences were seen in body wt or heart rate.

The responses to intraarterial norepinephrine at $0.06 \mu / \mathrm{kg}$ (Fig. 2) and lumbar sympathetic nerve stimulation at 10 and $20 \mathrm{~Hz}$ (Fig. 3) are illustrated. SHR showed significantly greater vascular responses to norepinephrine than did WKY. This vascular response was reduced, but not abolished by diazepam. Vasoconstrictor responses to lumbar sympathetic stimulation were also reduced in SHR by treatment with diazepam. Importantly, diazepam did not affect responses to norepinephrine or lumbar sympathetic stimulation in WKY.

Changes in vascular resistance resulting from the vasoactive agents epinephrine, angiotensin, barium chloride, and acetylcholine are summarized in Table 2. Vasoconstrictor responses were reduced in SHR-D. The vascular responses to acetylcholine were also reduced by diazepam. No reductions were observed in normotensive control diazepam treated animals (WKY-D).

\begin{tabular}{lcccc}
\multicolumn{5}{c}{ Table 1. Characteristics of $S H R$ and WKY treated with $D$ or $V^{1}$} \\
\hline
\end{tabular}

'Values represent means $\pm \mathrm{SE}$.

${ }^{2}$ Initial carotid artery pressure during hindquarter vascular resistance study.

${ }^{3}$ SHR-D is significantly less than SHR-V $(P<0.05)$. WKY groups are not significantly different from each other nor are they significantly different from SHR-D.

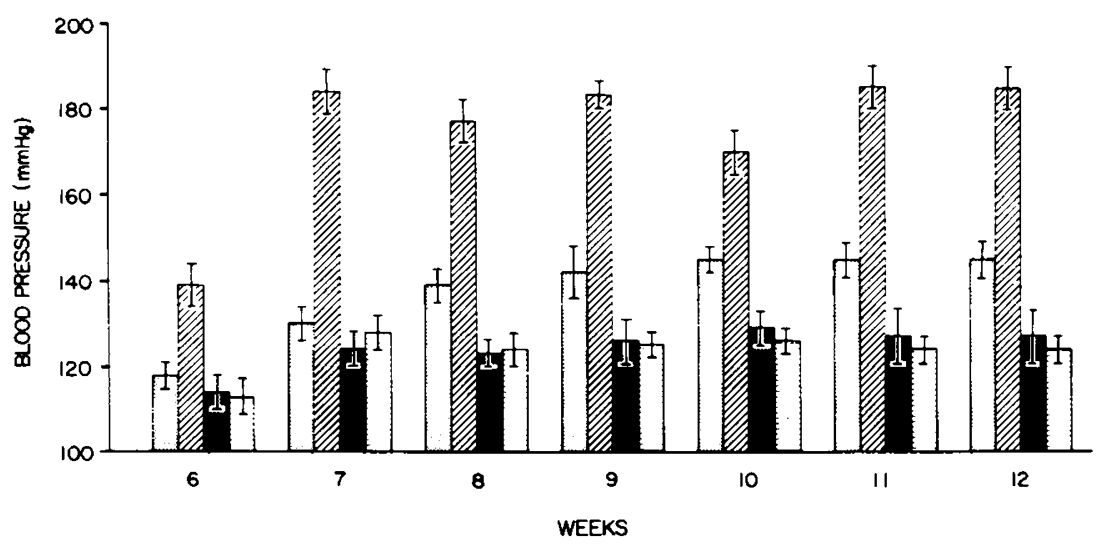

Fig. 1. Mean $\pm S E$ of systolic blood pressure measured by the tail cuff method from the 6th through the 12 th wk. The four histograms from left to right, each week, represent the following groups: 1) SHR-D; 2) SHR-V; 3) WKY-D; 4) WKY-V. SHR-D (leftward most histogram) are initially normotensive, but reach borderline hypertensive levels of $143 \mathrm{~mm} \mathrm{Hg}$. The blood pressure of SHR-V is distinctly different from the other groups at the 6th wk $(P<0.01)$ and remains different. No differences in blood pressure were observed throughout the course of the experiment for the normotensive WKY-V and WKY-D animals. By the 8th wk, the blood pressure of SHR-D was significantly different from both groups of the WKY animals, occupying an intermediate position between the distinctly hypertensive SHR-V and the normotensive control animals. 


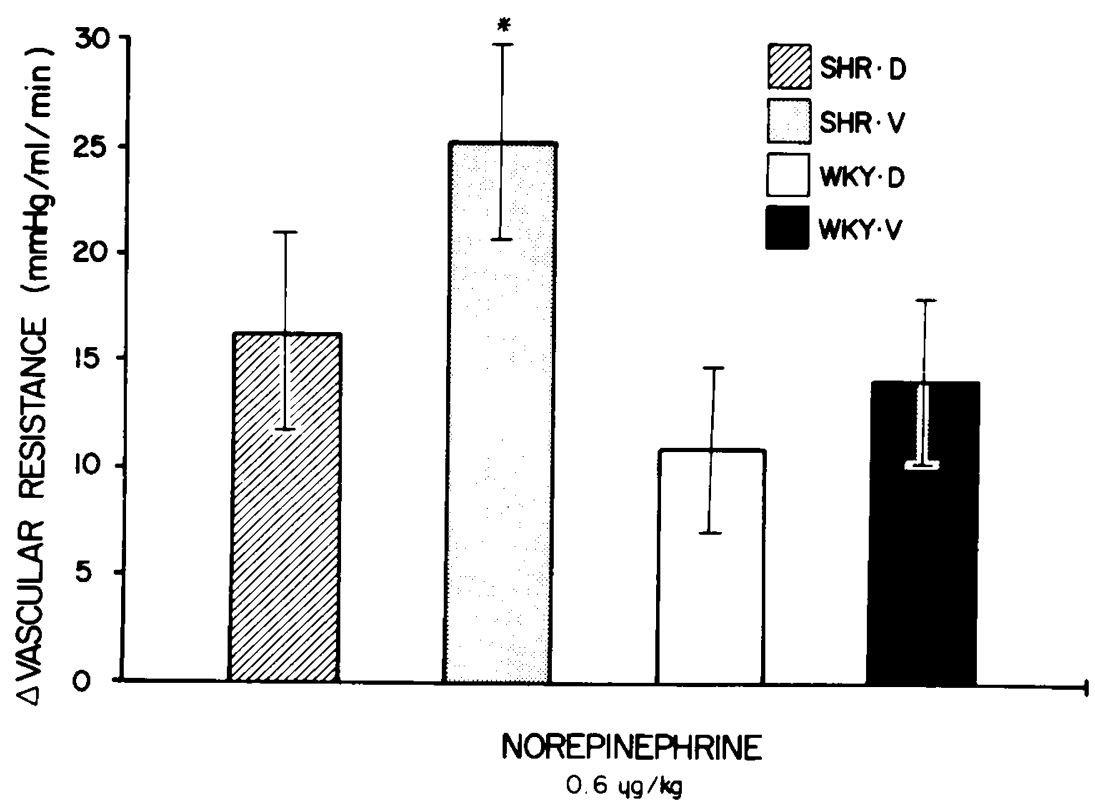

Fig. 2. The change in hindquarter vascular resistance (mean $\pm \mathrm{SD}$ ) to norepinephrine $(0.06 \mu \mathrm{g} / \mathrm{kg})$ in SHR and WKY treated with D or V. Responses in SHR-V were significantly greater than in SHR-D and both WKY groups. SHR-D were also significantly greater than both WKY groups.

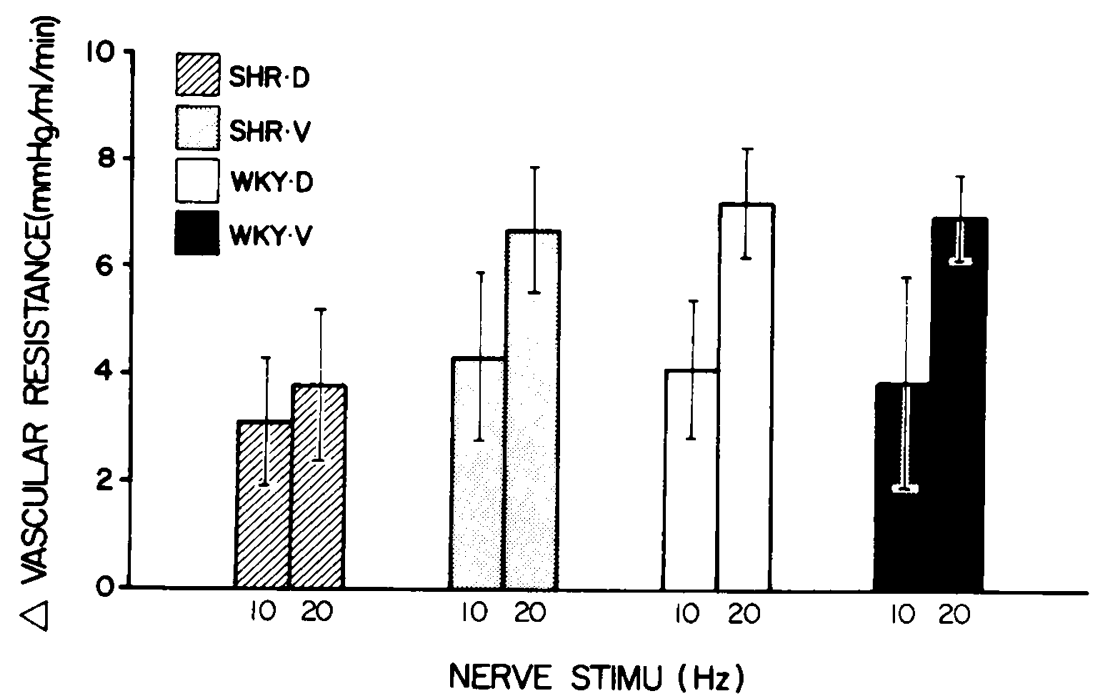

Fig. 3. The change in vascular resistance (mean $\pm \mathrm{SD}$ ) occurring with lumbar sympathetic nerve stimulation. At $20 \mathrm{~Hz}$, the response of SHR-D was significantly reduced as compared to SHR treated with vehicle SHR-V.

Table 2. Changes in vascular resistance produced by vasoactive agents in SHR and WKY treated with $D$ or $V^{1,2}$

$(\mathrm{mm} \mathrm{Hg} / \mathrm{ml} / \mathrm{min})$

\begin{tabular}{lcccc}
\cline { 2 - 5 } & $\begin{array}{c}\text { Epinephrine } \\
(0.06 \mu \mathrm{g})\end{array}$ & $\begin{array}{c}\text { Angiotensin } \\
(0.5 \mu \mathrm{g})\end{array}$ & $\begin{array}{c}\mathrm{BaCl}_{2} \\
(1.0 \mathrm{mg})\end{array}$ & $\begin{array}{c}\text { Acetylcholine }^{3} \\
(25 \mu \mathrm{g})\end{array}$ \\
\hline SHR-D & $19.1 \pm 5.3^{4}$ & $8.6 \pm 3.3^{4}$ & $6.6 \pm 3.6^{4}$ & $-16.2 \pm 1.4$ \\
SHR-V & $28.0 \pm 2.4$ & $17.20 \pm 2.6$ & $17.00 \pm 5.7$ & $-20.3 \pm 2.1$ \\
WKY-D & $12.7 \pm 2.4$ & $17.80 \pm 2.7$ & $16.40 \pm 4.7$ & $-45.6 \pm 3.3$ \\
WKY-V & $14.3 \pm 3.4$ & $16.20 \pm 2.4$ & $17.20 \pm 5.2$ & $-44.8 \pm 2.9$ \\
\hline
\end{tabular}

' Values represents means $\pm \mathrm{SE}$.

${ }^{2}$ All doses are per $\mathrm{kg}$ body weight.

${ }^{3}$ Percent of change from initial vascular resistance. Both SHR groups are significantly different $(P<0.05)$ from both WKY groups, but not from each other

${ }^{4}$ Responses in SHR were significantly reduced by diazepam $(p<0.01)$. Diazepam had no significant effect on responses in WKY.
In five adult SHR with established hypertension administered diazepam $1 \mathrm{mg} / \mathrm{kg}$ subcutaneously for 6 days, the blood pressure was not reduced by treatment $(183 \pm 23$ vs. $185 \pm 19 \mathrm{~mm} \mathrm{Hg})$ (Table 3). No significant differences in vascular reactivity were found between these animals and SHR-V.

\section{DISCUSSION}

Diazepam is a known psychotherapeutic agent of the 1, 4 benzodiazepine class whose chemical structure differs markedly from the commonly used antihypertensive drugs (15). Nonetheless, the chronic administration of diazepam from the newborn period prevented the full expression of hypertension in SHR. Treatment with diazepam reduced the blood pressure and vascular resistance to levels only slightly above those of the WKY group.

Diazepam treated SHR had diminished vascular responses both to stimulation of the lumbar sympathetic nerves and to the administration of the neurotransmitter substance, norepinephrine. 
Table 3. The effects of short term (6 day) administration of diazepam to adult $S H R$.

\begin{tabular}{lcr}
\hline & SHR-D & \multicolumn{1}{c}{ SHR-V } \\
\hline $\begin{array}{l}\text { Systolic blood pressure } \\
\quad(\mathrm{mm} \mathrm{Hg})\end{array}$ & $185 \pm 19$ & $178 \pm 20$ \\
Initial vascular resistance & $78 \pm 5$ & $76.5 \pm 6.0$ \\
$\quad(\mathrm{~mm} \mathrm{Hg} / \mathrm{ml} / \mathrm{min})$ & & \\
Norepinephrine $0.06 \mu \mathrm{g}^{1}$ & $29.0 \pm 2.8$ & $25.7 \pm 4.5$ \\
Epinephrine $0.06 \mu \mathrm{g}^{1}$ & $24 \pm 3$ & $28.0 \pm 2.4$ \\
Barium Chloride $1 \mathrm{mg}^{1}$ & $18.0 \pm 3.7$ & $17.0 \pm 5.7$ \\
Lumbar Sympathetic $20 \mathrm{~Hz}^{1}$ & $7.8 \pm 2.4$ & $6.7 \pm 1.2$ \\
Acetylcholine' $25 \mu \mathrm{g}$ & $-17 \pm 5.1$ & $-16.4 \pm 1.7$ \\
\hline
\end{tabular}

${ }^{1}$ Changes in vascular resistance $(\mathrm{mm} \mathrm{Hg} / \mathrm{ml} / \mathrm{min})$. All doses are per $\mathrm{kg}$ body wt.

In addition, diazepam reduced the vascular responses of SHR to potent vasoconstrictor agents such as angiotensin, epinephrine, and barium chloride. Vasoconstrictor responses, unlike most vasodilator responses, are independent and not directly proportional to the initial vascular resistance (6). A vasoconstricted vessel under high pressure might be expected to demonstrate a smaller percent of change in response to stimuli such as norepinephrine, tyramine, or sympathetic nerve stimulation. Nonetheless, the vasoconstrictor responses of untreated SHR to norepinephrine and lumbar nerve stimulation were steeper than those responses of the control animal, a response similar to that described by Lais et al. (14). Diazepam modified these enhanced responses of SHR to resemble more the responses of the normotensive control animal.

The vasodilation response of SHR to acetylcholine was reduced. Though vasodilator responses are usually proportional to the initial vascular resistance (23), Folkow et al. (4) demonstrated anatomic changes with the vessel walls of arteries subjected to chronic pressure increases, which uitimately interfere with the capacity of the vessel to dilate.

Diazepam may cause a loss of responsiveness to the neurotransmitter as opposed to depressing the release of transmitter from nerve terminals. Other data, however, argue against a specific neurotransmitter effect. The diminished vascular responsiveness of SHR after the chronic administration of diazepam to barium chloride, epinephrine, and angiotensin suggests a more generalized depression of vasoconstrictor responses, rather than a specific neurotransmitter effect. This diminished responsiveness was specific for diazepam treated SHR and was not observed in WKY animals.

The acute administration of diazepam to adult SHR with established hypertension produced neither a hypotensive effect nor reduction of vascular resistance, ruling out acute dosage as the total explanation. This implies that chronic administration begun in the newborn normotensive phase is critical. An early normotensive phase has been documented, as SHR do not appear to have elevated arterial pressure until 3-4 wk of age (13). Once the blood pressure is elevated, the hypertensive state is characterized by elevation of both systolic and diastolic pressure. Therefore, some unknown mechanism operates to transform the normotensive suckling SHR to the adult animal with severe hypertension. Though a central nervous system mechanism is not excluded, these data implicate the vascular smooth muscle as the common pathway to explain the diazepam induced reduction in blood pressure and resistance.

The cardiovascular actions of diazepam have been studied in the anesthetized, decerebrate cat (2). Diazepam causes a slight reduction in blood pressure, heart rate, and cardiac contractile force. The hypotensive action of iv diazepam $0.01 \mathrm{mg} / \mathrm{kg}$ in cat averaged $20 \mathrm{~mm} \mathrm{Hg}$, reaching a maximum in 2-3 min. The total duration of hypotension was 2-3 hrs. Both the hypothalamic and medullary pressor responses were blunted. The responses to stimulation of the stellate ganglia were unaltered despite iv doses of diazepam $6 \mathrm{mg} / \mathrm{kg}$. Those findings demonstrate that diazepam has a mild depressant effect upon the central nervous system cardiovasculatory centers, with the hypothalmus most affected.
The normal vascular adaptive mechanism to increased arterial pressure is medial hypertrophy (19). These structural changes are found in adult SHR (20). Folkow et al. (4) have demonstrated that raising the basal resistance to flow may contribute to the elevated vascular resistance observed in hypertension. In addition, abnormalities of responsiveness in SHR vessels have been documented (11). Three-wk-old SHR demonstrated vasoconstrictor hyperresponsiveness to norepinephrine at the time that hypertension was first detected. Though subtle structural changes may have already been present, they do not appear to adequately explain the specific effect of norepinephrine (12). Thus, the greatest increases in responsiveness were seen with catecholamines. Our data corroborate these exaggerated responses to catecholamines and show that after diazepam, the vascular responses of SHR to catecholamines, but not WKY, are depressed and become similar to those in untreated WKY. Because the responses to all vasoconstrictors are attenuated, the vascular depressant actions of diazepam appears to be nonspecific. Although the mechanism is not known, the effect is seen only with chronic administration and is great enough to attenuate markedly the expression of hypertension in SHR.

In conclusion, treatment from birth with diazepam exerts a profound effect in SHR upon the development of high blood pressure and elevated vascular resistance. The acute administration of diazepam to adult animals with established hypertension fails to reduce blood pressure or vascular resistance. Though these data do not rule out a central effect, the major effect of diazepam, most likely, is to cause a generalized depression of vascular smooth muscle responsiveness.

\section{REFERENCES AND NOTES}

1. Brody, M. J., Shaffer, R. A., and Dixon, R. L.: Method for the study of peripheral vascular responses in the rat. J. Appl. Physiol., 18: 645 (1963).

2. Chai, C. Y., and Wang, S. C.: Cardiovascular actions of diazepam in the cat. J. Pharm. Exptl. Ther., 154: 271 (1966).

3. Cowley, A. W., Liard, J. F., and Guyton, A. C.: Role of the baroreceptor reflex in daily control of arterial blood pressure and other variables in dogs. Circ. Res., 32: 564 (1973).

4. Folkow, B., Hallback, M., Lundgren, Y., Weiss, L.: Structurally based increase of flow resistance in spontaneously hypertensive rat. Acta. Physiol. Scand., 79. 373 (1970).

5. Fridman, M., and Freed, S.: Microphonic manometer for indirect determinations of systolic blood pressure in the rat. Proc. Soc. Exptl. Biol. Med., 70: 670 (1949).

6. Greenberg, S., and Wilson, W. R.: Lack of correlation between initial vascular resistance and responses to vasocontrictor stimuli in the perfused canine hindpaw. Proc Soc Exptl. Biol. Med., 145: 546 (1974).

7. Henry, J. P., Stephens, P. M., Axelrod, J.: Effect of psychosocial stimulation on the enzymes involved in the biosynthesis and metabolism of noradrenaline and adrenaline. Psychosom. Med., 33: 227 (1971).

8. Herd, J. A., Morse, W. H., Kelleher, R. T., Jones, L.: Arterial hypertension in the squirrel monkey during behavioral experiments. Am. J. Physiol., 217: 24 (1969).

9. Judy, W. V., Watanabe, A. M., Henry, D. P., Besch, H. R., Murphy, W. R. Hockel, G. N.: Sympathetic nerve activity: role and regulation of blood pressure in the spontaneously hypertensive rat. Circ. Res., (suppl. 2) 38: 21 (1976).

10. Lais, L. T., Bhatnager, R. K., and Brody, M. J.: Inhibition by dark adaption of the progress of hypertension in the spontaneously hypertensive rat (SHR). Proceedings of Council for High Blood Pressure Research. Circ. Res. (Suppl. 1) 34: 155 (1974).

11. Lais, L. T., and Brody, M. J.: Mechanism of vascular hyperresponsiveness in the spontaneously hypertensive rat, In: N. M. Kaplan: Hypertension XXIII: Hypertension Research, 1974 Clinical and Experimental. Circ. Res., (Suppl. 1) 36: 216 (1975)

12. Lais, L. T., and Brody, M. J.: Vacoconstrictor hyperresponsiveness: an early pathogenic mechanism in the spontaneously hypertensive rat. Eur. J. Pharmacol., 47: 177 (1978).

13. Lais, L. T., Rios, L. L., Boutelle, S., DiBona, G. F., and Brody, M. J.: Arterial pressure development in neonatal and young spontaneously hypertensive rats. Blood Vessels, 14: 277 (1977)

14. Lais, L. T., Shaffer, R. A., and Brody, J. J.: Neurogenic and humoral factors controlling vascular resistance in the spontaneously hypertensive rat. Circ. Res., 35: 764 (1974).

15. Medoff, H. S., and Bongiovanni, A. M.: Blood pressure in rats subjected to audiogenic stimulation. Am. J. Physiol., 143: 300 (1945).

16. Nosaka, S., Yamori, Y., Ohta, T., Okamoto, K.: Neural fraction of peripheral vascular resistance and vascular reactivity in the spontaneously hypertensive rat. In K. Okamoto: Spontaneous Hypertension: Its Pathogenesis and Complications. pp. 67-71 (Tokyo, Igaku Shoin, 1972). 
17. Okamoto, K., and Aoki, K.: Development of a strain of spontaneously hypertensive rats. Jap. Circ. J., 27: 282 (1963)

18. Randal, L. O., Heise, G. A., Shallek, W., Bagdon, R. E., Bansiger, R., Boris, A. Moe, R. A., and Abrams, W. B.: Pharmacological and clinical studies on valium, a new psychotherapeutic agent of the benzodiazepine class. Cur. Ther. Res., 3: 405 (1961).

19. Sivertsson, R.: The hemodynamic importance of structural vascular changes in essential hypertension. Acta. Physiol. Scand., (Suppl.) 343: 1 (1970)

20. Suwa, N., Takahashi, T.: Morphological and morphometrical analysis of circulation in hypertension and ischemic kidney. (Munchen-Berlin-Wein, Urban and Schwarzenberg, 1971).

21. Thomas, C. B.: Experimental hypertension from section of moderator nerves. Bull. Johns Hopkins Hospital, 74: 335 (1944).

22. Tukey, J. W.: Comparing individual means in the analysis of variance. Biometrics, 5: 99 (1949).
23. Wilder, J.: Stimulus and Response: The Law of Initial Values. (Bristol, Wright, 1967).

24. Yamori, Y., Matsumoto, M., Yamabe, H., and Okamoto, K.: Augmentation of spontaneous hypertension by chronic stress in rats. Jap. Circ. J. 33: 399 (1969).

25. This research was supported, in part, by a program project grant from the University of Iowa College of Medicine \#HLB-14388 and a Biomedical Research Support Grant RR 05372 from the Biomedical Research Support Branch, Division of Research Facilities and Resources, National Institutes of Health.

26. Requests for reprints should be addressed to: Richard M. Schieken, M. D. Department of Pediatrics, Division of Pediatric Cardiology, University of Iowa Hospitals and Clinics, lowa City, IO 52242 (USA)

27. Received for publication March 28, 1978.

28. Accepted for publication August 24, 1978 